\title{
FIR CHANNEL IDENTIFICATION IN MULTIRATE COMMUNICATION SYSTEMS WITH A SUBSPACE METHOD
}

\author{
Hongbo Yan and Sumit Roy \\ Dept. of Electrical Engineering \\ University of Washington Box 352500 \\ Seattle, Washington 98195
}

\begin{abstract}
The problem of blind channel identification for multirate, multi-user communication systems is addressed. By exploiting symbol rate differences, it is shown that users can be separated based on the autocorrelation of the received signal, thus reducing the problem to the familiar single rate setting. A subspace method is then developed to identify the channel associated with each user. Simulations are used to explore algorithm performance as a function of key factors such as signal to noise ratio (SNR) and signal to interference ratio (SIR).
\end{abstract}

\section{INTRODUCTION}

Blind channel identification continues to be of active research interest because it facilitates equalization and improves bandwidth efficiency. Either explicitly or implicitly, the early blind methods exploited higher order statistics (HOS), see [2]. The primary disadvantage of HOS-based approaches was the need for large number of samples for accurate estimation of the relevant higher-order statistics. The seminal work of [4] showed that for wide-sense cyclostationary signals, blind channel identification was possible based only on secondorder statistics (SOS). This inspired many subsequent SOS-based methods for blind identification, described in the recent review [3].

The extensive literature to date on SOS-based blind identification however exclusively concentrates on single rate systems. Next (3rd) generation personal communication systems must accommodate heterogeneous traffic, such as voice, video and data that are inherently multirate. Accordingly, receiver design issues for multirate systems is expected to be of increasing importance as evidenced by a growing body of work [6]. However, channel estimation approaches and their per-

This work was supported in part by in part by AFOSR Grant F 49620-1-0472 formance evaluation for multi-user, multirate systems remains relatively open.

In this paper, we develop a subspace method for blind estimation of channels for multirate communication systems. The approach utilizes the signal cyclostationarity features and is based on the autocorrelation function of the sampled received signal. By exploiting the differences in user periodicity in the autocorrelation function resulting from respective symbol (baud) rates, we extract the component due to each user. A subspace method is then devised to estimate the unknown channel parameters for individual users.

\section{PROBLEM STATEMENT}

A generic multirate communication system operating over finite duration multipath channels is shown in Fig. 1. The mathematical description is given by [1]

$$
h_{i}(t)=\sum_{j=1}^{L_{p}} \alpha_{j} c_{i}\left(t-\tau_{j}\right)=\left(\sum_{j=1}^{L_{p}} \alpha_{j} \delta\left(t-\tau_{j}\right)\right) \odot c_{i}(t)
$$

where $c_{i}(t)$ is the transmit pulse shape for the $i$-th user, and $\alpha_{i}(t)=\sum_{j=1}^{L_{p}} \alpha_{j} \delta\left(t-\tau_{j}\right)$ represents a $L_{p^{-}}$ path physical channel with respective delays $\tau_{j}$ for the $j$-th path and $\odot$ denotes convolution. The baseband received signal is given by

$$
y(t)=\sum_{i=1}^{K} \sum_{k=-\infty}^{\infty} s_{i}(k) h_{i}\left(t-k T_{i}\right)+w(t)
$$

where $s_{i}(k)$ 's are mutually independent zero-mean i.i.d. input sequences with variance $\sigma_{i}^{2}, K$ is the number of distinct symbol rates and $w(t)$ is additive white Gaussian noise with variance $\sigma_{w}^{2}$. Note that we assume for simplicity that there is only one user at each rate in the system, therefore the term $i$-th user will refer to rate $i$ user in the following. Without loss of generality, 


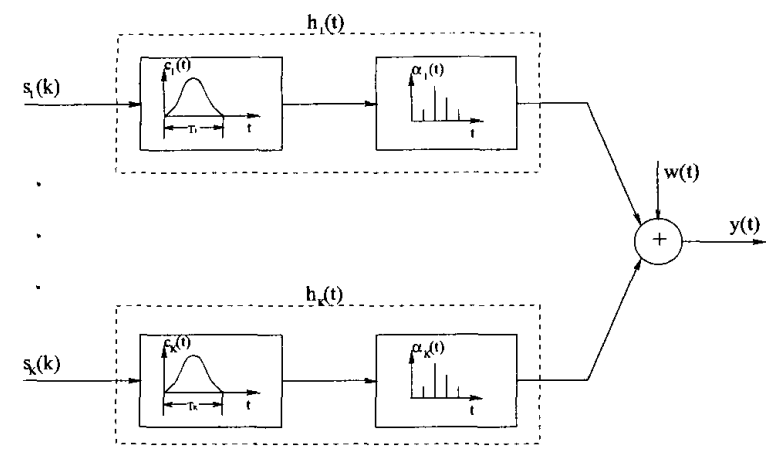

Figure 1: A multirate communication system

suppose the ratio between these rates is

$$
\frac{T_{1}}{p_{1}}=\frac{T_{2}}{p_{2}}=\cdots=\frac{T_{K}}{p_{K}}=T
$$

where $p_{1}, p_{2}, \cdots, p_{K}$ are co-prime integers. We denote $\frac{1}{T}$ as the basic rate. The received signal is oversampled with respect to the basic rate by a factor $\Delta=\frac{T}{L}$ to yield

$$
y(n \Delta)=\sum_{i=1}^{K} \sum_{k=-\infty}^{\infty} s_{i}(k) h_{i}\left(n \Delta-k T_{i}\right)+w(n \Delta)
$$

resulting in the following discrete time model

$$
y(n)=\sum_{i=1}^{K} \sum_{k=-\infty}^{\infty} s_{i}(k) h_{i}\left(n-k L p_{i}\right)+w(n),
$$

The problem addressed in this paper is the blind estimation of $h_{i}(n)$ based on $y(n)$.

\section{OUTLINE OF APPROACH}

The algorithm proposed in this section exploits the cyclostationarity of the oversampled received signal. Let $y_{i}(k)=\sum_{k=-\infty}^{\infty} s_{i}(k) h_{i}\left(n-k L p_{i}\right)$ be the component of $y(n)$ due to the $i$-th user; its autocorrelation is given by

$$
\begin{aligned}
& r_{y_{i}}[n, m]=E\left\{y_{i}(n) y_{i}^{*}(n-m)\right\} \\
& =\sigma_{i}^{2} \sum_{k=-\infty}^{\infty} h_{i}\left(n-k L p_{i}\right) h_{i}^{*}\left(n-m-k L p_{i}\right)
\end{aligned}
$$

And the autocorrelation of the noise-free received signal is

$$
r_{y}[n, m]=E\left\{y(n) y^{*}(n-m)\right\}=\sum_{i=1}^{K} r_{y_{i}}[n, m]
$$

The following are easily verifiable:

Result 1: $r_{y_{i}}[n, m]$ is a periodic function in $n$ with fundamental period $\mathcal{P}_{i}=L p_{i}$.

Result $2 r_{y}[n, m]$ is periodic in $n$ with fundamental period $\mathcal{P}=L p_{1} p_{2} \cdots p_{K}$ and

Result 3: $\tilde{r}_{y_{i}}[n, m]=r_{y}[n, m]-r_{y_{i}}[n, m]$ is periodic in $n$ with fundamental period $\mathcal{Q}_{i}=L \mathcal{P} / \mathcal{P}_{i}$.

Thus, for any $n_{1}=j_{1} \mathcal{Q}_{i}+d, n_{2}=j_{2} \mathcal{Q}_{i}+d$, where $j_{1}, j_{2}, d$ are integers with $0 \leq d \leq \mathcal{Q}_{i}-1$ we have

$$
\begin{aligned}
& r_{y}^{n_{1}, n_{2}}(m)=r_{y}\left[n_{1}, m\right]-r_{y}\left[n_{2}, m\right] \\
& =\left(r_{y_{i}}\left[n_{1}, m\right]+\tilde{r}_{y_{i}}\left[n_{1}, m\right]\right)-\left(r_{y_{i}}\left[n_{2}, m\right]+\tilde{r}_{y_{i}}\left[n_{2}, m\right]\right) \\
& =\left(r_{y_{i}}\left[n_{1}, m\right]-r_{y_{i}}\left[n_{2}, m\right]\right)+\left(\tilde{r}_{y_{i}}\left[n_{1}, m\right]-\tilde{r}_{y_{i}}\left[n_{2}, m\right]\right) \\
& =r_{y_{i}}\left[n_{1}, m\right]-r_{y_{i}}\left[n_{2}, m\right] \\
& =r_{y_{i}}^{n_{1}, n_{2}}(m)
\end{aligned}
$$

where the fourth equation follows from Result 3.

Lemma: For $n_{1}, n_{2}$ satisfying $n_{1} \bmod \mathcal{Q}_{i}=n_{2} \bmod \mathcal{Q}_{i}$, $r_{y}^{n_{1}, n_{2}}(m)=r_{y_{i}}^{n_{1}, n_{2}}[n, m]$ establishes the key user separation result.

\subsection{The Algorithm}

Denote $f_{i}^{l}(m)=\sum_{k=-\infty}^{\infty} h_{i}(m) \delta\left(m+k \mathcal{P}_{i}-l\right), g_{i}(m)=$ $h_{i}^{*}(-m)$ and assume $\sigma_{i}^{2}=1$ for simplicity. Thus (6) can be rewritten as

$$
r_{y_{i}}[n, m]=f_{i}^{n}(m) \odot g_{i}(m)
$$

and

$$
r_{y_{i}}^{n_{1}, n_{2}}(m)=\left(f_{i}^{n_{1}}(m)-f_{i}^{n_{2}}(m)\right) \odot g_{i}(m)
$$

It follows from its periodicity that $r_{y_{i}}^{n_{1}, n_{2}}(m)$ is not identically zero if $n_{1} \bmod \mathcal{P}_{i} \neq n_{2} \bmod \mathcal{P}_{i}$ and at the same time $f_{i}^{n_{1}}(m) \neq f_{i}^{n_{2}}(m)$ for some $m$. Hence (8) indicates that if $n_{1}$ and $n_{2}$ further satisfy $n_{1} \bmod \mathcal{Q}_{i}$ $=n_{2} \bmod \mathcal{Q}_{i}$, then $r_{y}^{n_{1}, n_{2}}(m)$ are nonzero statistics only about the $i$-th user. Denoting $q_{1}=n_{1} \bmod \mathcal{P}_{i}, q_{2}=$ $n_{2} \bmod \mathcal{P}_{i}$, we search over $\left(n_{1}, n_{2}\right)$ such that $n_{1} \bmod \mathcal{Q}_{i}=$ $n_{2} \bmod \mathcal{Q}_{i}$ for all $\left(q_{1}, q_{2}\right)$ pairs satisfying $q_{1}<q_{2}$. We index all these $\left(q_{1}, q_{2}\right)$ pairs as $1,2, \cdots, J_{i}$. Note that it suffices to only consider $0 \leq n_{1}, n_{2} \leq \mathcal{P}-1$ because of the periodicity of $r_{y}^{n_{1}, n_{2}}(\mathrm{~m})$ and $q_{1}<q_{2}$ because $\left(q_{1}, q_{2}\right)$ and $\left(q_{2}, q_{1}\right)$ are essentially the same. Accordingly in the following we replace $r_{y}^{n_{1}, n_{2}}(m), r_{y_{i}}^{n_{1}, n_{2}}(m)$ with $r_{y}^{(l)}(m), r_{y_{i}}^{(l)}(m)\left(1 \leq l \leq J_{i}\right)$ respectively. Introducing $f_{i}^{(l)}(m)=f_{i}^{n_{1}}(m)-f_{i}^{n_{2}}(m)$, we obtain the following compact version of (10) involving the observation statistics and the unknown channel parameters $h_{i}(n)$

$$
r_{y}^{(l)}(m)=r_{y_{i}}^{(l)}(m)=f_{i}^{(l)}(m) \odot g_{i}(m)
$$


Denote $\mathbf{r}_{y}(m)=\left[\begin{array}{lll}r_{y}^{(1)}(m) & \cdots & r_{y}^{\left(J_{i}\right)}(m)\end{array}\right]^{T}, \mathbf{f}_{i}(m)=$ $\left[\begin{array}{lll}f_{i}^{(1)}(m) & \cdots & f_{i}^{\left(J_{i}\right)}(m)\end{array}\right]^{T}$, suppose $h_{i}(n), \mathbf{f}_{i}(m)$ have support $\left[\begin{array}{ll}0 & N_{i}\end{array}\right]$ and define:

$$
\mathbf{F}_{i}^{T}=\left[\begin{array}{cccc}
\mathbf{f}_{i}(0) & \cdots & \mathbf{f}_{i}\left(N_{i}\right) & \\
\ddots & \ldots & \ldots & \ddots \\
& \mathbf{f}_{i}(0) & \cdots & \mathbf{f}_{i}\left(N_{i}\right)
\end{array}\right]_{M_{i} J_{i} \times\left(M_{i}+N_{i}\right)}
$$$$
\mathbf{G}_{i}^{T}=\left[\begin{array}{cccc}
g_{i}\left(-N_{i}\right) & \cdots & g_{i}(0) & \\
\ddots & \ldots & \cdots & \ddots \\
& g_{i}\left(-N_{i}\right) & \cdots & g_{i}(0)
\end{array}\right]
$$$$
\mathbf{R}_{i}^{T}=\left[\begin{array}{cccc}
\mathbf{r}_{y}\left(-N_{i}\right) & \ldots & \mathbf{r}_{y}\left(N_{i}\right) & \\
\ddots & \ldots & \ldots & \ddots \\
& \mathbf{r}_{y}\left(-N_{i}\right) & \cdots & \mathbf{r}_{y}\left(N_{i}\right)
\end{array}\right]_{M_{i} J_{i} \times\left(M_{i}+2 N_{i}\right)}
$$

Now (10) yields a matrix form equation:

$$
\mathbf{R}_{i}=\mathbf{G}_{i} \mathbf{F}_{i}
$$

Result: If $\mathbf{F}_{i}$ has full row rank, $\mathbf{R}_{i}$ and $\mathbf{G}_{i}$ share the same column space, which can be determined by an appropriate subspace revealing decomposition. Applying the Singular Value Decomposition (SVD) to the $\left(M_{i}+2 N_{i}\right) \times M_{i} J_{i}$ block Toeplitz matrix $\mathbf{R}_{i}$ yields

$$
\mathbf{R}_{i}=\mathbf{G}_{i} \mathbf{F}_{i}=\left(\begin{array}{ll}
\mathbf{U}_{s} & \mathbf{U}_{o}
\end{array}\right)\left(\begin{array}{cc}
\boldsymbol{\Lambda}_{s} & \mathbf{0} \\
\mathbf{0} & \mathbf{0}
\end{array}\right)\left(\begin{array}{c}
\mathbf{V}_{s}^{H} \\
\mathbf{V}_{o}^{H}
\end{array}\right)
$$

where $\mathbf{U}_{s}$ spans the $\left(M_{i}+N_{i}\right)$-dimensional signal subspace defined by the columns of $\mathbf{G}_{i}$, and $\mathbf{U}_{o}$ spans its orthogonal complement, i.e.

$$
\mathbf{G}_{i} \perp \mathbf{U}_{o} \Rightarrow \mathbf{G}_{i}^{H} \mathbf{U}_{o}=\mathbf{0}
$$

Exploiting the Toeplitz structure of $\mathrm{G}_{i},(14)$ can be translated into

$$
\mathcal{U}_{o}^{T} \mathbf{h}_{i}=\mathbf{0}
$$

where

$$
\begin{gathered}
\mathbf{h}_{i}=\left[\begin{array}{lll}
h_{i}\left(N_{i}\right) & \cdots & h_{i}(0)
\end{array}\right]^{T} \\
=\left[\begin{array}{ccc}
g_{i}(-N) & \cdots & g_{i}(0)
\end{array}\right]^{H} \\
\mathcal{U}_{o}=\left[\begin{array}{ccc}
\mathbf{U}_{o}(1,:) & \cdots & \mathbf{U}_{o}\left(M_{i}+N_{i},:\right) \\
\mathbf{U}_{o}(2,:) & \cdots & \mathbf{U}_{o}\left(M_{i}+N_{i}+1,:\right) \\
\vdots & \ddots & \vdots \\
\mathbf{U}_{o}\left(N_{i}+1,:\right) & \cdots & \mathbf{U}_{o}\left(M_{i}+2 N_{i},:\right)
\end{array}\right]
\end{gathered}
$$

with $\mathbf{U}_{o}(m,:)$ being the $m$-th row vector of $\mathbf{U}_{o}$. From different viewpoint, in [7] Qiu et. al. used this method to compute the greatest common divisor (GCD) of several polynomials. Denoting $\mathbf{Q}=\mathcal{U}_{o}^{*} \mathcal{U}_{o}^{T}$, the channel vector can be identified up to a scalar in the presense of noise through optimization

$$
\hat{\mathbf{h}}_{i}=\arg \min _{\left\|\mathbf{h}_{i}\right\|=1}\left\|\mathbf{h}_{i}^{H} \mathbf{Q} \mathbf{h}_{i}\right\|^{2}
$$

\subsection{Identifiability}

As elaborated in the previous subsection, channel identifiability with our subspace method requires that $\mathbf{F}_{i}$ have full row rank. A necessary condition for this is that it has fewer rows than columns, i.e. $M_{i}+$ $N_{i}<M_{i} J_{i}$. Whenever $J_{i} \geq 2$, this necessary condition can always be satisfied by choosing an appropriate $M_{i}$. Then the identifiability theorem in [5] applies to the new multirate scenario because $\mathbf{F}_{i}^{T}$ has the same structure as that of the filtering matrix (also called generalized Sylvester matrix) in [5].

Theorem 1 The channel $h_{i}(n)$ can be uniquely determined from (15) up to a complex scalar iff

1) for $0 \leq n_{1}, n_{2} \leq \mathcal{P}-1$, the total number of $\left(q_{1}, q_{2}\right)$ pairs $J_{i} \geq 2$ where $q_{1}=n_{1} \bmod \mathcal{P}_{i}, q_{2}=n_{2} \bmod \mathcal{P}_{i}$ such that $q_{1}<q_{2}$ and $n_{1} \bmod \mathcal{Q}_{i}=n_{2} \bmod \mathcal{Q}_{i}$;

2) the polynomials $\mathbf{F}_{i}^{(l)}=\sum_{n=0}^{N_{i}} f_{i}^{(l)}(n) z^{-n}, \quad l=$ $1, \cdots J_{i}$, do not share any common zeros.

\section{SIMULATION RESULTS}

We considered a dual-rate system with $p_{1}=2, p_{2}=3$ and $L=2$, implying $\mathcal{P}_{1}=4, \mathcal{P}_{2}=6$ and $\mathcal{P}=12$. Following the user seperation condition, we choose $(0,2)$, $(1,3)$ as $\left(q_{1}, q_{2}\right)$ pairs for the 1st user and $(0,2),(0,4),(1,3)$, $(1,5),(2,4),(3,5)$ as $\left(q_{1}, q_{2}\right)$ pairs for the 2 nd user. $r_{y}^{(l)}(m)$ are then evaluated according to (8) from the corresponding $\left(n_{1}, n_{2}\right)$ pairs. The sample autocorrelation is computated through

$r_{y}[n, m]=\frac{1}{N_{h}-N_{l}+1} \sum_{j=N_{l}}^{N_{h}} y[n+j \mathcal{P}+m] y^{*}[n+j \mathcal{P}]$

where $N_{l}=\max (0,\lceil-n / \mathcal{P}\rceil,\lceil-(n+m) / \mathcal{P}\rceil), N_{h}=$ $\min \left(\left\lfloor\left(N_{o}-n\right) / \mathcal{P}\right\rfloor,\left\lfloor\left(N_{o}-n-m\right) / \mathcal{P}\right\rfloor\right)$ with $\lceil x\rceil(\lfloor x\rfloor)$ standing for the smallest (greatest) integer that is greater than or equal to (less than or equal to) $x$ and $N_{o}$ representing the length of observation sequence.

The performance of the proposed algorithm is assessed by normalized root mean-square error (NRMSE), defined as

$$
N R M S E=\frac{1}{\|\mathbf{h}\|} \sqrt{\frac{1}{N_{t}} \sum_{q=1}^{N_{t}}\left\|\hat{\mathbf{h}}_{q}-\mathbf{h}\right\|^{2}}
$$


where $\hat{\mathbf{h}}_{q}$ is the channel estimate at the $q$ th trial, $\mathbf{h}$ is real channel and $N_{t}$ is the total number of Monte-Carlo trials, which is 100 in our simulations.

We tested the two-ray multipath channel

$$
h_{i}(t)=\lambda_{1} p_{i}\left(t-\gamma_{1} T_{i}\right)+\lambda_{2} p_{i}\left(t-\gamma_{2} T_{i}\right) \quad i=1,2
$$

where $\lambda_{1}, \lambda_{2}$ are zero-mean complex Gaussian random variables with unit variance in each component (real and imaginary). The path delays parameters $\gamma_{1}, \gamma_{2}$ are random variables uniformly distributed on $[-11] . p_{i}(t)$ is the raised-cosine pulse shaping function with roll-off factor 0.5 and time limited to $4 T_{i}$. The same set of $\lambda_{l}, \gamma_{l}$ $(l=1,2)$ parameters were used for both channels.

With data samples received over the duration of $500 p_{2} T_{1}=500 p_{1} T_{2}$. (i.e. 1500 symbols at rate 1,1000 symbols at rate 2 ), we obtained the NRMSE curves shown in Fig. 2 and 3. Fig. 2 indicates robustness of the proposed algorithm against multirate interference, i.e., the effectiveness of our user seperation scheme. It is also seen that the first user outperforms the second one from this perspective. In the presense of noise, the method still works successful. However the relative performance of the two users changes in spite of the fact that at high SNR the first user still has lower NRMSE. Eextensive simulations show that the channel order is an important factor that results in this behavior. In our case, the second user has a longer channel duration than the first one.

\section{REFERENCES}

[1] J. Proakis, Digital Communications, 3rd ed. New York: McGraw Hill, Inc., 1995.

[2] J. Mendel, "Tutorial on higher-order statistics (spectra) in signal processing and system theory: Theoretical results and some applications," proc. IEEE, vol.79, no.3, pp.278-305, Mar. 1991.

[3] L. Tong and S. Perreau, "Multichannel blind channel estimation: from subspace to maximum likelihood methods," Proc. IEEE, vol.86, no.10, pp.1951-1968, Oct. 1998.

[4] L. Tong, G. Xu and T. Kailath, "Blind identification and equalization based on second order statistics: a time-domain approach," IEEE Trans. Infom. Theory, vol.40, no.2, pp.340-349, Mar. 1994.

[5] E. Moulines, P. Duhamel, J. Cardoso and S. Mayrargue, "Subspace methods for the blind identification of multichannel FIR filters," IEEE Trans. Signal Processing, vol.43, no.2, pp.516-525, Feb. 1995.

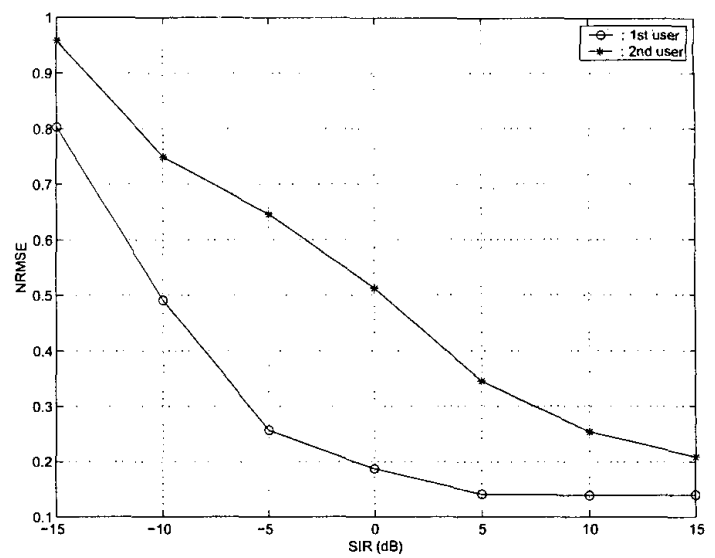

Figure 2: NRMSE versus SIR: noise free

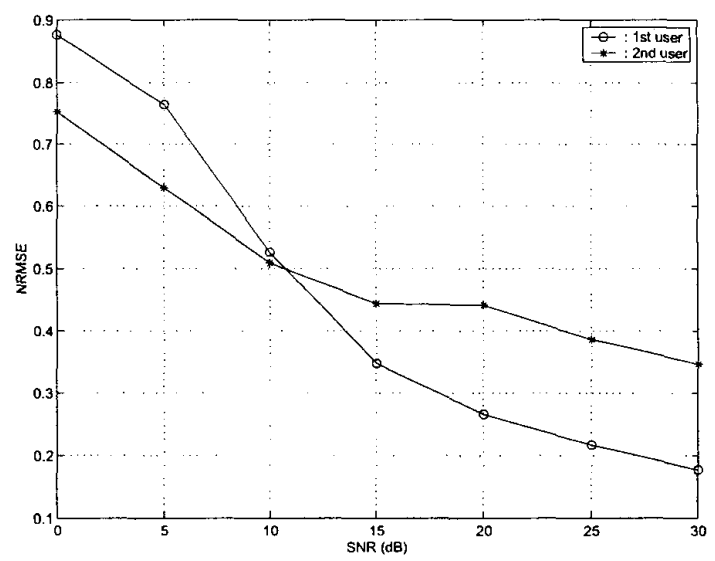

Figure 3: NRMSE versus SNR: $\mathrm{SIR}=5 \mathrm{~dB}$

[6] M. L. Honig and S. Roy, "Multi-user communication with multiple symbol rates," in Proc. IEEE Int. Symp. on Infom. Theory, Whistler, BC. Canada, pp.381, Sept. 1995.

[7] W. Qiu, Y. Hua and K. Abed-Meraim, "A subspace method for the computation of the GCD of polynomials", in Automatica, vol.33, no.4, pp.741743, April 1997. 